\title{
Synchrotron X-ray fluorescence analysis in environmental and earth sciences
}

\author{
F. Adams ${ }^{\mathrm{a}}$ \\ Department of Chemistry, University of Antwerp, 2610 Wilrijk, Belgium
}

\begin{abstract}
Compared to other microscopic analytical tools X-ray microscopy techniques have the advantage that the large penetration depth of X-rays in matter allows one to investigate the interior of an object without destructive sample preparation. In combination with X-ray fluorescence tomography, analytical information from inside of a specimen can be obtained. Different X-ray analytical techniques can be used to produce contrast, X-ray absorption, fluorescence, and diffraction, to yield chemical, elemental, and structural information about the sample. Scanning microscopy on the basis of various lens systems in synchrotron radiation sources provides a routine spatial resolution of now about 100 nanometer but in the foreseeable future a 10-20 nanometer spatial resolution can be expected. X-ray absorption spectrometry can also provide chemical (speciation) information on the sample. All this makes X-ray microscopy attractive to many fields of science. In this paper the techniques are briefly reviewed and a number of applications in the earth, planetary and cosmos sciences are illustrated with state-of-the art examples, while applications in the environmental sciences and biology are also briefly discussed.
\end{abstract}

\section{Introduction}

X-ray spectrometry (XRS) provides well established, non-destructive analytical methods that can be applied in a large variety of fields of application (e.g., materials science, quality control, environmental science, geology, life science and archaeometry). X-ray fluorescence spectrometry (XRF) is an elemental analysis method that works at ambient air pressure and does not require complicated sample preparations [1,2]. It has been widely used for qualitative and quantitative analyses. Micro-XRF analysis $(\mu$-XRF) has been used as powerful tool for the acquisition of elemental images of X-ray fluorescence through the use of X-ray focusing techniques. The technique can be applied in the laboratory - e.g. with poly-capillary X-ray optics - but it takes a long time to obtain elemental images using the scanning method if a large area is measured with high spatial resolution. With high brilliance synchrotron radiation sources images can be obtained more rapidly while simultaneously increasing the sensitivity by orders of magnitude. Synchrotrons are accelerator-based sources of exceptionally intense, tightly focused beams of X-rays and ultraviolet radiation, as well as infrared, that make possible both basic and applied research in fields ranging from physics to biology to technology that are not possible with more conventional laboratory-type equipment.

Synchrotron radiation (SR) is the specific electromagnetic radiation generated in storage rings from high energetic electrons. Since its discovery in high-current accelerators for particle physics there use rapidly evolved to specific machines to produce X-ray beams.

\footnotetext{
a e-mail: Freddy.Adams@ua.ac.be
}

This is an Open Access article distributed under the terms of the Creative Commons Attribution-Noncommercial License 3.0, which permits unrestricted use, distribution, and reproduction in any noncommercial medium, provided the original work is properly cited. 
Synchrotron radiation has a number of unique properties for the purpose of X-ray analysis: brightness is many orders of magnitude more intense than that from any conventional X-ray tubes and radiation is highly collimated as SR is emitted in a narrow cone in the forward direction tangential to the electron orbit. Synchrotron light is emitted with energies ranging from infrared light to hard $(>50 \mathrm{keV}) \mathrm{X}$-rays and is tunable. Its radiation is also highly polarized. Especially the linear polarization in the SR ring plane is of interest here as it can considerably reduce background radiation, hence, increase detection limits.

More than 50 synchrotron light sources have been built up to the present that can be used by researchers on experimental areas (beamlines) designed for specific application areas. A number of new facilities are now in construction or in the planning phase [3].

Large storage rings introduced in the late 1980 s were specifically designed to optimize SR brightness. These -so-called- third generation storage rings were specifically designed to optimize SR brightness. The resulting X-ray beams are increasingly used as powerful microscopes with high spatial resolution in sensitive elemental, structural and molecular analysis using diffraction, phase contrast, absorption, fluorescence, ...). The most important of these storage rings are the European Synchrotron Radiation Facility (ESRF) in Grenoble $(6 \mathrm{GeV})$, the Advanced Photon Source (APS) at Argonne National Laboratory, near Chicago (7 GeV) and the SPring-8 in Japan $(8 \mathrm{GeV})$.

Several high performance soft X-ray synchrotron sources have been operating for a number of years such as, for Europe ELETTRA in Italy and BESSY in Germany. At the hard X-ray end of the spectrum with energies above $50 \mathrm{keV}$ the only European source which can rival the ESRF is PETRAIII in Germany where in 2009 the first monochromatic X-ray beam was extracted and end of June 2010 five beamlines were in the commissioning phase. Nationally operated European medium energy light sources are SOLEIL in France, DIAMOND in the United Kingdom, SLS in Switzerland, MAX IV in Sweden and ALBA in Spain [3].

This infrastructure at SR facilities offers possibilities to do research at a number of specific beamlines. The ESRF for instance has in excess of 6,000 user visits each year to perform experiments at its forty-three beamlines. The public beamlines that are built and exploited by the ESRF make use of insertion devices, mostly undulators and provide optimized X-radiation for a wide range of applications. A number of beamlines are also exploited by user groups (collaborative research groups, CRGs) at the bending magnets of the storage ring.

This brief review deals with the methodology and the applications of SR sources for trace-level analysis of materials on the microscopic and sub-microscopic scale. Most natural environmental materials are heterogeneous, so it is useful to have analytical techniques with spatial resolution sufficient to resolve heterogeneities in both composition and structure.

This paper can be considered as an update of an earlier now largely outdated contribution on the same topic in the same series that was published in 1999 [4]. More general information on X-ray techniques for microanalysis is available, e.g. in Janssens et al. [5] and Adams [6].

Elemental and molecular analysis with XRS is described in some detail. Micro-XRF, which is an important technique for the analysis of small areas, is one of the significant trends in XRS. Two dimensional and three dimensional methods of analysis are discussed in their quantitative aspects. Related methods of analysis based on absorption edge phenomena such as X-ray absorption spectrometry (XAS) provide molecular information. Computerized XRF micro-tomography (XFCT) based on the penetrative character of X-rays gives 3D information of small samples. Microscopic X-ray diffraction (XRD) provides structural data on the sample and is also briefly discussed.

\section{X-ray spectrometry}

The strength of X-ray micro-analytical techniques compared to other analytical methodologies depends on a number of favourable circumstances. The large penetration depth of high energetic $\mathrm{X}$ rays in matter allows one to investigate the interior of an object without destructive sample preparation. Thus in combination with tomography, local information from inside of a specimen can be obtained, even from inside special non-ambient sample environments. Different X-ray interaction and 
measurement conditions can be used to produce contrast, such as X-ray absorption, fluorescence, and diffraction, to yield chemical, elemental, and structural information about the sample, respectively.

Within the range of "beam" analytical techniques, X-rays represent by far the least destructive way to perform chemical imaging. This makes X-ray microscopy attractive to many fields of science, ranging from physics and chemistry to materials, geo-, and environmental science, biomedicine, and nanotechnology. The penetration of X-rays in matter also implies that contrarily to the vacuum requirements of most other microprobe methods, samples can normally be observed in the ambient environment allowing the measurement of samples in their natural (e.g. in wet) conditions. This is a particular advantage and often a prerequisite for many environmental and biological applications.

\subsection{Synchrotron X-ray spectrometry}

The analytical performance of XRS analysis depends critically on the intensity of the X-ray source. Synchrotron radiation sources have enormous advantages in comparison to more conventional sources.

A particular advantage of SR X-ray analytical techniques is the extremely high source brilliance (number of photons emitted per unit source area over a unit angle of emission and per unit energy, photons $\mathrm{s}^{-1} \mathrm{mrad}^{-2} \mathrm{~mm}^{-2}$ per $0.1 \%$ of radiation bandwidth). As such SR is ideally suited for the generation of focused X-ray beams with very high intensity, exceeding now well over $10^{10}$ photons $\mathrm{s}^{-1} \mu \mathrm{m}^{-2}$ in a spot size of now down to well below 100 nanometer.

Highly-monochromatic X-ray microbeams can be generated through the use of X-ray monochromators. By tuning the energy of the beam, the strong energy dependence of the inner shell photoelectric cross sections can then be exploited to either increase measurement specificity or to obtain chemical-state information on elements of interest (speciation). In the plane of the storage ring the generated radiation is also linearly polarized. This polarization of the incident radiation can be used to reduce the relative contribution of scattered radiation reaching the detector (scattering cross sections are dependent on the polarization whereas the photo absorption cross sections and fluorescence radiation is not). This increases considerably the signal-to-background ratios (by up to 2 orders of magnitude) compared to non-polarized conditions (out of the plane defined by the storage ring).

Micro-XRF is a rapidly evolving analytical technique which allows visualising the trace level elemental distributions within a specimen in an essentially non-destructive manner. At second generation SR sources, detection limits at the sub-parts per million concentration level can be obtained with micrometer resolution, while at third generation sources the spatial resolution can be better than 100 nanometer and sensitivities approach the attogram $\left(10^{-18} \mathrm{~g}\right)$ level. Optimal detection limits are illustrated in Fig. 1 for the state-of-the-art ESRF ID22NI nanoprobe station with a beam size of approximately $100 \mathrm{~nm}$, beam intensity of $6 \times 10^{11} \mathrm{ph} / \mathrm{s}$ and measuring times per individual data point of $3 \mathrm{~s}$ and $1,000 \mathrm{~s}$.

\subsection{X-ray focusing}

The most obvious way to achieve nanoscale resolution in imaging is to scan a nanometer-sized beam across the sample (further on we will see that there are alternatives). The spatial resolution is then defined by the beam size. Demagnification (focusing) for the formation of intense focused X-ray microbeams is not obvious when considering the complex index of refraction:

$$
\mathrm{n}=1-\delta-\mathrm{i} \beta \text {. }
$$

In this equation $\delta$ is the refractive index decrement (typically $10^{-6}$ for hard $\mathrm{x}$-rays) and $\beta$ is the absorption index. The complex refractive index of all materials is very close to unity and less than one, which makes the construction of refractive lenses quite difficult especially when one also considers the strong radiation absorption of any material from which the focusing element is made. Despite of this, demagnification of a quite unidirectional X-ray beam from a SR source is now readily available using various types of X-ray optics. The high directionality and brightness of SR beams is important for the realization of the full focusing potential. 


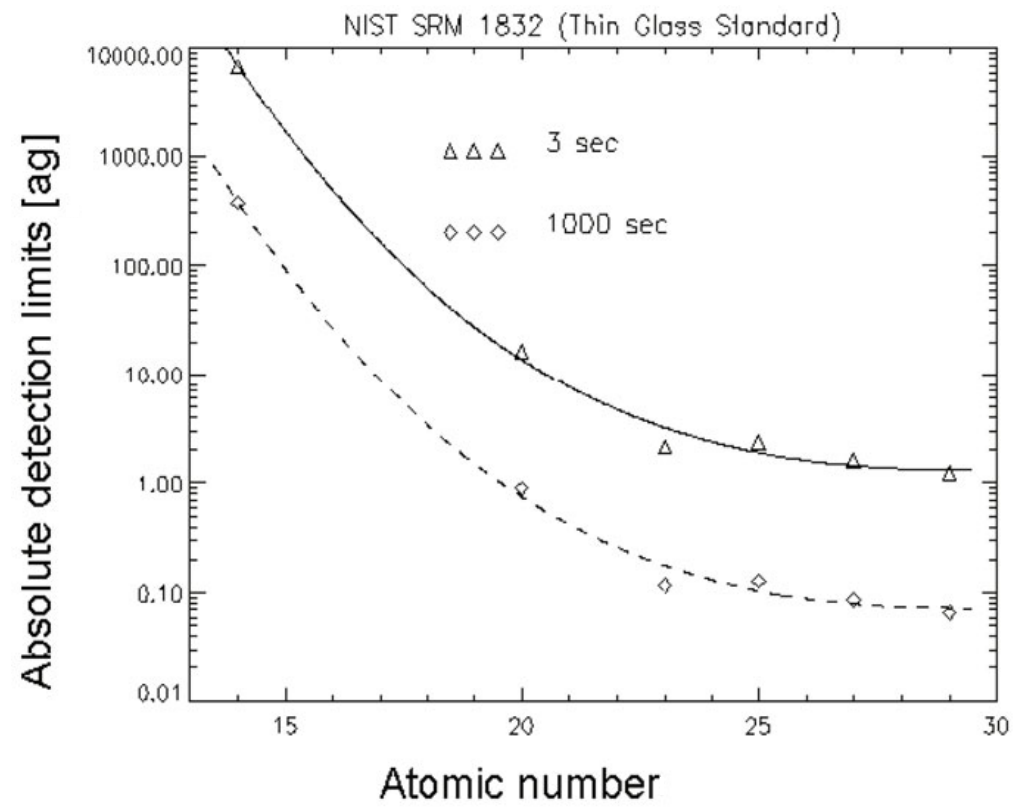

Fig. 1. Absolute detection limits for the ESRF ID22NI nanoprobe station with a beam size of approximately 100 nanometer, beam intensity of $6 \times 10^{11} \mathrm{ph} / \mathrm{s}$ and measuring times of $3 \mathrm{~s}$ and $1000 \mathrm{~s}$.

In general, there are three principles in optics; "refraction", "reflection", and "diffraction". Optic that focus electromagnetic radiation by employing refraction are "lenses" while optics that employ reflection are "mirrors". In the visible and infrared part of the electromagnetic spectrum, all three principles are commonly employed. For example, different wavelengths can be dispersed by a "prism" using refraction, and by a "grating" using diffraction.

Bent mirrors, crystals and multi-layers, tapered glass mono-capillaries, complex poly-capillary lens systems, Bragg-Fresnel lenses, Fresnel zone plates (FZP) lenses, one or two dimensional waveguides and refractive lenses have been developed and tested for use in micron size to sub-micron focusing at synchrotron beam lines [5,6]. Focusing hard X-rays with the help of grazing-incidence on reflecting surfaces is the method of choice used on many SR beamlines. The goal to decrease the spot size to a micrometer or less has triggered technological developments of a specific much used reflecting and focusing system, the so-called Kirkpatrick-Baez (K-B) system. Two orthogonal mirrors focus the beam successively in the horizontal and in the vertical planes.

Glass poly-capillary optics relies on single or multiple reflections at very shallow angle. They are simple to manufacture and easy to use in focusing optics. Such achromatic optics has acceptances of several $\mathrm{mm}$ horizontally and vertically, while their angular acceptances can be matched to the source divergence by the design of the poly-capillary lens [7]. The technology for constructing refractive lenses is rapidly improving by using nanofabrication techniques. Unfortunately, since the index of refraction depends on the X-ray energy, refractive lenses are chromatic and this substantially limits their applicability.

At present, it is possible to obtain sufficient beam intensity on microscopic samples to allow reliable sub ppm even ppb level determinations of a large number of elements. Many optical devices have been developed to concentrate X-ray beams to very small spots, sometimes even smaller than 100 nanometer. A far limit achieved up to now is the successfully production of a nanometer sized X-ray beam with a width of 7 nanometer at $20 \mathrm{keV}$ [8]. This focal spot was obtained using a laterally graded multi-layer mirror and a grazing-incidence deformable mirror.

Monochromatic radiation can be obtained at high energy resolution $\left(\Delta \mathrm{E} / \mathrm{E} \sim 10^{-4}\right)$ and high flux $\left(10^{11} \mathrm{ph} / \mathrm{s}\right.$ with double crystal K-B monochromators. This resolution is necessary for scanning around absorption edges in XAS applications. For straightforward XRF measurements for elemental analysis 
$\Delta \mathrm{E} / \mathrm{E} \sim 10^{-2}$, as obtained typically with a multi-layer monochromator, is sufficient and provides a considerably higher radiation flux.

\subsection{D and 3D imaging}

When performing XRF measurements over a cross-section of a sample that is much larger than the beam itself, by systematically translating the sample with respect to the beam, a 2-dimensional image of the various elements detectable in the X-ray spectra is obtained. As the primary beams penetrate relatively deeply below the surface of the samples in such 2D XRF images a depth averaged signal is obtained. In XRF tomography (XFCT) this disadvantage is turned into an advantage and a 3-dimensional stack of elemental images is obtained.

For full XRF tomography, repetitive linear scans at a selected height in the sample are recorded for different rotation angles over $360^{\circ}$ rotation. The different line profiles, as a function of the rotation angle, form so-called elemental sinograms. The fluorescence images can then be reconstructed from the combination of the data obtained, yielding elemental image cross-sections at a well-defined height of the sample. To reach the third dimension, one has to shift the sample vertically and repeat the entire process at different heights until the full 3-dimensional image of the sample is obtained.

One of the main limitations associated with the XFCT technique is the lengthy data collection procedure required; obtaining a detailed $3 \mathrm{D}$ image of an object takes a long measurement time of several hours on a SR beamline. A lot of progress has been realized recently in data collection speed. The main limitation for improving overall measurement speed is due to detector capabilities in data throughput in order to prevent overload of the detection system - rather than the X-ray beam intensity. A set-up with two detectors placed at $180^{\circ}$ to the rotation axis, can reduce the measurement time by a factor of 2 (by reducing the rotation from $360^{\circ}$ to $180^{\circ}$ ). Charge Coupled Device (CCD) detectors are used in laboratory type absorption computerized tomography but are not available for fluorescence analysis.

Obtaining quantitative information is not a trivial task considering the immense amount of data and the need to correct for absorption and secondary fluorescence of the X-rays in the sample. Apart form the computerized tomography procedures there is the need to deconvolute the (often quite noisy) X-ray spectra and to apply correction for absorption and fluorescence of the X-rays in the sample. The use of chemometric tools is needed for the interpretation of the data. Spectral deconvolution by non-linear least squares [9] and quantification models based on Monte Carlo simulation software were developed in the author's laboratory and published in a series of papers in the period 1993-2000 [e.g. [10-13]]. Thanks to the full understanding of the X-ray interaction process in matter, computer techniques, particularly those based on Monte Carlo simulation, are able to predict the spectral response without limitations in approximations or idealizations of the sample geometry [14]. Hence, they allow calibration without the need to recur to standard reference materials and correction for radiation absorption, thus opening up the way to perform reliable quantitative analysis at accuracy levels of the order of $<20 \%$.

\subsection{Confocal XRF}

An alternative to XRF tomography approach consists in the application of confocal XRF. Confocal excitation/detection uses a poly-capillary half-lens in front of the energy dispersive detector and provides direct analysis of specific locations in the beam path below the sample. We refer to Fig. 2 for the principle of the technique, when using two poly-capillary focusing elements. For systematic measurements over the three spatial dimensions the method provides 3D analysis in a more direct way (there is no need for image reconstruction) than in full XFCT [15].

This method provides a suitable alternative to XFCT for the detailed analysis of specific well defined volumes within larger samples and is also called local fluorescence tomography. Instead of the need to scan the full sample cross-section of a large sample, including areas of no interest, it allows mapping of a specific predetermined microscopic region of interest with high image resolution within a larger object (e.g. an inclusion). 


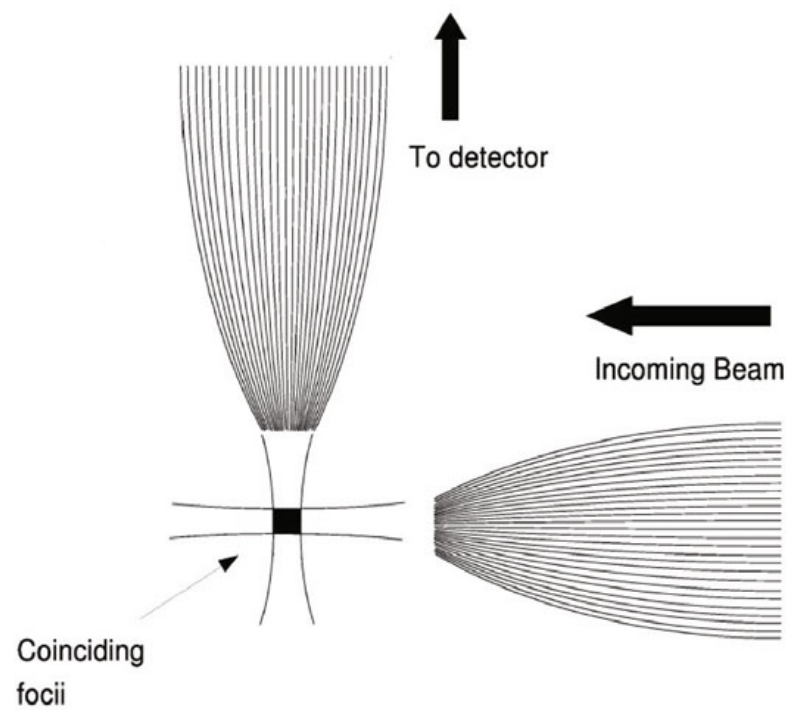

Fig. 2. Geometry of confocal analysis with two poly-capillary lenses for focusing the incoming radiation and the emitted radiation to the detector (B. De Samber, PhD thesis, reference [46]).

\subsection{X-ray absorption edge spectroscopy}

Chemical (speciation) analysis through energy scanning around the $\mathrm{K}$ or L edge at high energy resolution $\left(\Delta \mathrm{E} / \mathrm{E} \sim 10^{-4}\right.$ or higher) is now possible at the spatial resolution level in the nanoscopic observation domain well below the $\mu \mathrm{m}$. The fine structure of X-ray absorption edges in X-ray absorption spectroscopy (XAS) depends on the local chemical environment and state of the excited atom. Measurement in the transmission or the fluorescence modes provides this chemical information with two complementary methods, X-ray absorption near edge structure (XANES) and extended X-ray absorption fine structure analysis (EXAFS).

Normally standard XAS experiments are performed in transmission or fluorescence detection mode with millimeter size X-ray beams. However, in various research disciplines, including the environmental sciences, earth and planetary sciences, cultural heritage studies etc., samples are, in general, not homogeneous and analysis with an X-ray beam with micrometer dimensions is then necessary. When a microscopically inhomogeneous sample is measured with such beam dimensions, an average spectrum over the illuminated area is obtained in which the information on the spatial differences is lost. To obtain spatially resolved spectra of microscopically heterogeneous samples, it is necessary that the focus is maintained while scanning over the energy range of interest.

The part of the spectra covered by XANES extends from the pre-edge region to approximately $50 \mathrm{eV}$ above the absorption edge. In the pre-edge region some features may be visible due to electron transitions to empty, or partially filled, bound excited states [16]. The higher energy part of a XAS spectrum above the edge is the EXAFS region which provides oscillations generated by the constructive and destructive interferences between the outgoing and backscattered photoelectron wave and provides information on characteristics of the neighbouring atoms and their coordination number.

A wide variety of samples can be studied with XAS: gases, solids, liquids, crystalline and amorphous materials, bulk layers, surfaces, adsorbed atoms, and interfaces. Due to the penetrating character of X-rays (of high enough energy) through gases and liquids, XAS can be applied for in situ studies in catalysis, during layer growth, and phase transitions, etc. The technique is also increasingly used in environmental studies.

\subsection{Total reflection X-ray fluorescence}

In total reflection X-ray fluorescence (TXRF), minute amounts of sample are excited at very low angle of incidence by the X-ray standing wave field generated above a reflector surface, due to interference of 
incoming and reflected beams $[17,18]$. An important attribute of this measurement condition is that the penetration depth of the X-ray field inside the sample substrate becomes very small thereby drastically reducing the spectral background, which in turn improves the detection sensitivities by several orders of magnitude.

TXRF is mainly used for non-destructive surface- contamination analysis in the semiconductor industry and for chemical-trace analysis of impurities in water or other liquids after they are evaporated on a clean flat surface.

The technique offers limits of detection (LDs) in the picogram range for excitation with X-ray tubes and even in the femtogram region when SR is used for the analysis of environmental particles. The technique can also be applied in combination with XANES. For instance, size-fractionated aerosol samples were collected on silicon wafers using a seven-stage cascade impactor and were analyzed by means of SR-TXRF at beamline L at HASYLAB, Hamburg, Germany [19]. This approach allowed the quantitative determination of ultra-trace concentrations (at the level of picogram $/ \mathrm{m}^{3}$ ). The use of the technique for the analysis of micro/nano size particles is also possible (see further). SR-TXRF can also be used in combination with angle-dependent measurements (X-ray standing beam spectroscopy, XSW) and XANES to gain additional structural information about the investigated sample.

\subsection{Micro X-ray diffraction}

In addition to the measurements of compositional differences over a heterogeneous object, micro-X ray diffraction (micro-XRD) is becoming a common tool on many X-ray microprobes. Micro-XRD, the punctual and systematic measurement of X-ray diffraction patterns provides information on the variation of its crystallographic structure. The XRD maps can be obtained together with the maps of elemental information and can assist considerably in the characterization of heterogeneous objects. To a certain extent XRD and XAS are complementary techniques as they both provide information on the local neighbourhood of the atoms..

Highly heterogeneous samples of fly-ash particles have been analyzed at a microscopic level by simultaneously recording XRF intensities and X-ray diffraction patterns. From the data obtained the main minerals in the particles could be identified in the different particle size fractions [20].

\subsection{Coherence imaging}

Most of the X-ray images recorded up to now were collected on the basis of absorption contrast. In terms of the complex X-ray refractive index (equation $1, n=1-\delta-\mathrm{i} \beta$ ) this means that the emphasis is placed on the imaginary component $\mathrm{i} \beta$. In phase contrast imaging (PCI) we are concerned with the $\delta$ term of the equation. PCI exploits the coherence of radiation at $3^{\text {rd }}$ generation synchrotron sources and X-ray Free-Electron Lasers (FEL) and opens the possibility to exploit imaging at an unprecedented spatial resolution level within a sample. PCI methods often rely on a relatively large object-to-detector distance to allow wave interference (Fresnel diffraction) to occur and manifest itself as contrast in the image plane.

It is possible to obtain 3D density maps of the sample (holo-tomography). These techniques are explored at present and will require substantial instrumental and theoretical developments (model reconstruction) to reach maturity [21]. PCI will involve a combination of phase and absorption contrast to varying degrees and while both generally decrease with increasing X-ray energy, it is important to note that $\delta$ varies as $\lambda^{2}$ whereas $\beta$ varies as $\lambda^{4}$ (with $\lambda$ the X-ray wavelength), in the absence of any elemental absorption edges. Hence the effects of phase contrast become progressively more dominant, relative to absorption-contrast effects, at higher X-ray energies.

Phase-contrast X-ray imaging provides substantially enhanced contrast resolution for soft-tissues compared to conventional absorption radiography. Although it is essentially a visualization technique, not an analytical tool, it can be of interest for pinpointing extremely small areas of interest in heterogeneous samples. For example, extremely small aerosol-induced lung injuries were observed by PCI [22]. 


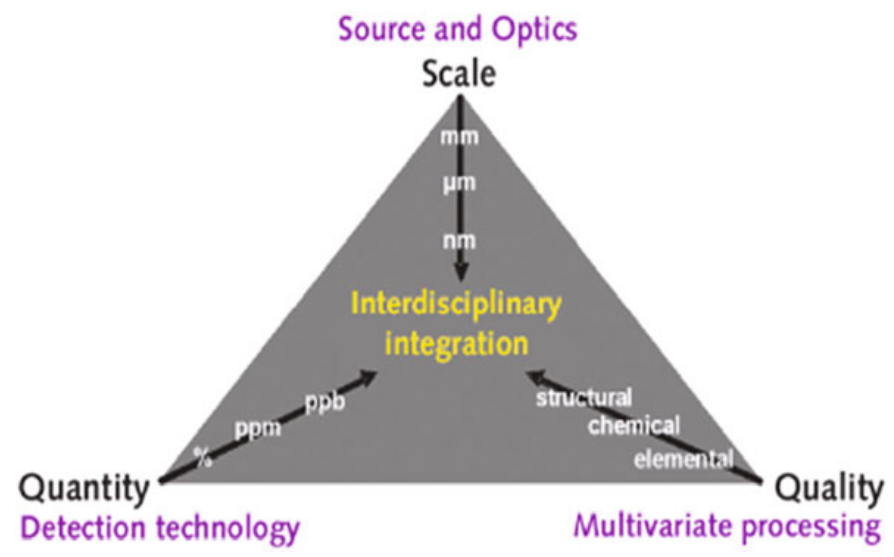

Fig. 3. Synchrotron radiation-based microanalytical techniques provide multi-scale information and progress in performance on the image level, the detection limits and the quality level of information (adapted from Bouvet et al., reference [21]).

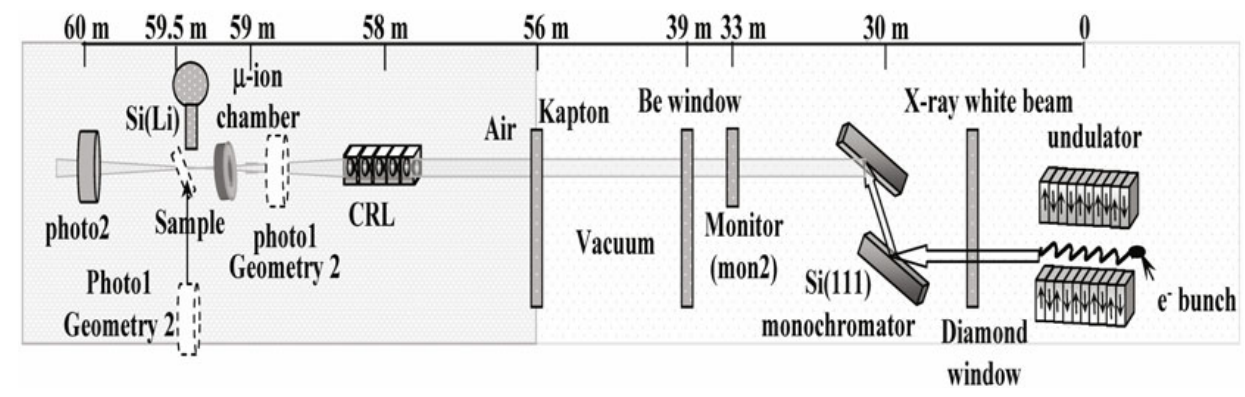

Fig. 4. Schematic layout of the ID18 beamline and the ID18F user end-station. The photodiode 1 (photo 1) is either placed into the sample position if a sample was measured (geometry 1 ) or in front of the mini-ionization chamber (geometry 2) that records the input flux for calibration (adapted from Somogyi et al., reference [28]).

\section{State of the art and future prospects}

At present there are quite a large number of SR installations that offer beamlines for imaging XRS techniques as research tool for a wide user community working in the earth-, environmental-, and life-sciences. They offer advanced imaging techniques in multimodal mode, i.e. a range of techniques such as those discussed above are simultaneously available. The SR techniques complement laboratory based instrumentation based on XRS or other beam techniques. The demand for beam time is such that the use is limited to important and non-routine applications for which other analytical tools cannot provide the required information. There is a rapidly growing interest in imaging and analysis and new instruments are designed pushing the application in versatility, sensitivity and imaging resolution. Figure 4 illustrates the areas of advances [21].

In what follows we will give two examples of future prospects, one in a new nationally operated mid-energy European SR source and one at the ESRF. In addition we also give a brief survey of two set-ups for analysis that are available since a number of years at the ESRF.

\subsection{The nanoscopium beamline at SOLEIL}

Nanoscopium is the single scanning hard X-ray nano-probe beamline that is being built at the SOLEIL storage ring near Gif-sur-Yvette, France. This $\sim 155 \mathrm{~m}$ long beamline will fully exploit the high brilliance and coherence characteristics of the X-ray beam both for diffraction limited focusing and for contrast formation for earth-, environmental-, and life-sciences. The different micro and nano-probe 
techniques offered by the beamline will permit elemental mapping at trace (ppm) levels with scanning XRF, speciation mapping with XANES, phase gradient mapping (scanning differential phase contrast), and density-contrast based imaging of internal structures (coherent diffraction imaging) in the 30 nanoeterm to $1 \mu \mathrm{m}$ spatial resolution range, in "in situ conditions". The beamline will cover the $5-20 \mathrm{keV}$ energy range. K-B mirror and FZP lenses are used as focusing devices. The beamline is foreseen to be open for users at the beginning of 2013 [23].

\subsection{The ESRF upgrade programme}

The ESRF storage-ring light source was built in the early 1990s and an Upgrade Programme was decided in 2008 [21]. This upgrade programme is highlighted as one of thirty-five infrastructure projects identified on the European Strategy Forum for Research Infrastructures (ESFRI) 2006 Roadmap [24].

The ESRF upgrade aims at advancing innovative X-ray-based methodologies and puts special emphasis on imaging techniques and their combination with X-ray scattering and spectroscopic techniques. The upgrade includes development of several nanofocus beamlines enabling hard X-ray nanoprobes with 50 to 20 nanometer beam size. The design features recognize that there is a growing interest for scientific research at the interfaces between classical disciplines which are expected to grow rapidly over the next few years. With its emphasis on the development of nano-science the environmental sciences and earth and planetary sciences will fully benefit from the ESRF Upgrade Programme as it will provide a unique portfolio of highly specialised instruments via the development of new beamlines.

The coherence of X-ray beams opens the possibility to exploit phase contrast imaging to observe detail at unprecedented levels within a sample. 3D X-ray holo-tomography based on phase contrast was employed in the ESRF for the applications of X-ray micro-tomography in a non-destructive 3D studies of paleontological specimens e.g. to look inside precious opaque amber resin containing fossilised insects and spiders from the Carboniferous period [25].

\subsection{Spatially resolved micro-XANES at the DUBBLE XAS station of the ESRF}

In addition to the new initiatives that try to push limits of performance in sensitivity and spatial resolving power there are a number of existing installations that make steady progress in performance. We give one example at the Dutch-Belgian beamline (DUBBLE, BM26A), a CRG beamline at the ESRF that provides micro-EXAFS and XANES at a standard 3rd generation bending magnet. The incoming beam is focused using a poly-capillary half-lens and a second glass poly-capillary is placed in front of the energy dispersive X-ray detector to establish the confocal detection geometry with a design as in Fig. 2. With this poly-capillary based set-up transmission efficiencies of 25-45\% with flux density gain factors of about 2,000 and beam sizes of 10-20 micrometer are obtained in the 7-14 keV energy range. The full-width-half-maxima along the main axes of the resulting ellipsoidal detection volume are $18.5 \times 12.0 \times 10.0$ micrometer $^{3}$ at the $\mathrm{Cu}$ K-edge. The confocal micro-XANES mode was applied in the 3D resolved study of mineral inclusions in rare natural diamonds at the Fe K-edge [26].

\subsection{The ID18F micro-XRF beamline at the ESRF}

Originating in a collaborative effort between the ESRF and the author's laboratory at the University of Antwerp, the ID18F microprobe was designed to be a stable instrument with prominence given to the ease of use, reliability and repeatability of the system. A primary aim of the end-station was to develop micro-XRF as an accurate and traceable quantitative analytical tool at the microscopic level and as a reference tool for other (laboratory-type) micro-analytical techniques. In addition to these high accuracy XRF microprobe measurements, the instrument is a multi-technique station which can offer simultaneous focused-probe fluorescence and wide or small angle X-ray scattering measurements and the possibility for fluorescence tomography and absorption tomography acquisitions [27]. 
A typical set-up for analytical microanalysis is shown in Fig. 4. With an energy range of 6-28 keV, a monochromacity of $\sim 10^{-4} \Delta \mathrm{E} / \mathrm{E}$, a flux of $5.10^{10}$ photons/s and possibilities for focusing down to $1.5 \times 4$ micrometer $^{2}$, it is an instrumentation that is optimally suited for environmental and earth science applications.

\section{Examples of applications in environmental and earth sciences}

A brief review including a few characteristic recent applications will be given to illustrate the possibilities of the different methodology discussed. It includes the analysis of sediments and atmospheric particles, fly-ash particles, applications in the earth sciences (analysis of diamond and fluid inclusions, 3D determinations in microscopic extraterrestrial micro-objects). Apart from all this, there is a growing interest in the biomedical community in obtaining information concerning the distribution and local chemical environment of metals in tissues and cells [29] on which we give one example in section 4.6.

\subsection{Elemental imaging of single particles}

The environmental impact of fly ash particles is basically influenced by the chemical composition of the particle surface. The interaction of the particle with the atmospheric, soil, and biological environment occurs through leaching in the water phase. The leachability of different elements depends on the elemental and structural composition of the particles in the interacting phases. In order to understand the interaction processes, the difference in chemical composition as a function of depth below the surface of trace and minor elements in individual particles is of importance. Micro XRF is a suitable technique for the study of the particles either with laboratory instrumentation or with SR sources. The penetration of energetic X-rays of $15-30 \mathrm{keV}$ in fly ash is several orders of magnitude larger than that of protons in an alternative approach using proton induced X-ray emission analysis (PIXE) [30].

When a SR microprobe is used to study the distribution of trace elements in fly ash, the particles must be made available as thin sections. The thickness of these should be similar to the diameter of the exciting beam in order to avoid blurring of the elemental image. On the other hand, the excited beam needs to irradiate a minimum sample mass in order to obtain a detectable signal for low amounts of analytes of typically the order of 10 femtogram.

In addition to bulk analytical applications there are numerous possibilities for single particle characterization [31]. We will just mention one example that illustrates the state-of-the-art that concerns the analysis of radioactive particles.

The mobility, biological uptake and accumulation of radionuclides are essentially dependent on the physico-chemical form of the nuclides associated with particles. To obtain information on particle characteristics such as particle size distribution, structure, elemental and radionuclide particle composition as well as oxidation state of matrix elements, solid-state speciation techniques are needed $[32,33]$. Electron microscopy as well as microscopic XRS techniques utilising SR radiation have proved most useful in providing such information. Different high energy microanalysis techniques (micro-XRF, micro-XANES, micro-XRD) were combined to characterise individual micrometer sized radioactive particles originating from the nuclear weapons accident in Thule, Greenland in 1968 [34]. It was shown that particle characteristics including weathering rates and mobilisation of associated radionuclides are source specific and release-scenario dependent. Fuel particles released during the explosion are characterised by $\mathrm{UO}_{2}$-cores with surrounding laver of reduced $\mathrm{U}$ with low weathering rate. The results demonstrated that $\mathrm{U}$ and $\mathrm{Pu}$ are homogeneously distributed throughout the particles, indicating that $\mathrm{U}$ and $\mathrm{Pu}$ have been fused. Furthermore, micro-XANES showed that $\mathrm{U}$ and $\mathrm{Pu}$ in the particles are present as mixed oxides. $\mathrm{U}$ is found to be in oxidation state IV whereas Pu apparently is a mixture of $\mathrm{Pu}$ (III) and $\mathrm{Pu}$ (IV) [34].

The same techniques were applied oil single depleted Uranium (DU) particles to link the potential bioavailability of these particles to site-specific particle characteristics. The oxidation states and crystallographic forms of U in DU particles were determined for individual particles isolated from selected samples collected at different sites in Kosovo and Kuwait that were contaminated by DU ammunition during the 1999 Balkan conflict and the 1991 Gulf war [35]. 
A pluridisciplinary approach was used to study iodine immobilization mechanisms by biocarbonates in a natural marine carbonate-bearing clay formation. Different techniques of observation (optical microscope, scanning electron microscopy and electron microprobe analysis, infrared spectrometry, spatially resolved SR micro XRF and micro XRD) were used on carbonate shells of the Callovian-Oxfordian (160 Ma) clay formation from the ANDRA (French Radioactive Waste Management Agency) Underground Research Laboratory (Meuse/Haute Marne, France). The combined results indicate that the biostructure of the Rhynchonella shell is relatively well-preserved but bioaragonite slowly transforms into calcite, whereas the bivalve shell is entirely recrystallized into diagenetic calcite and celestite. Electron microscopy and micro-XRF data also show bioaccumulation of iodine in carbonate shells [36].

\subsection{Three-dimensional trace-element analysis of diamond inclusions}

Inclusions in sub-lithospheric natural diamonds provide direct information on the physical and chemical conditions in the deep Earth, down to the upper interface of the lower mantle. Confocal microbeam XRF can be used to obtain so-far inaccessible in situ information on the composition of the mineral phases present in these inclusions in diamonds that are kept in their original (i.e., unopened) form. This includes the determination of fully three-dimensional distributions of minor and trace elements within these closed systems to obtain detailed information of the composition in all phases present [26].

Earth's deep convecting upper mantle is believed to represent a rather homogenous geochemical reservoir of spinel or garnet lherzolite with primitive major element and moderately depleted trace element composition. Only where subduction occurs is this homogeneity disrupted by a suite of rocks ranging from eclogites/garnet pyroxenites (former oceanic crust) to residual harzburgites. In addition to these peridotitic and metabasaltic rocks results show the presence of a chemically distinct reservoir in the deep convecting upper mantle. Indeed, in situ structural analyses (micro-XRD and microRaman spectroscopy) and three-dimensional trace element mapping (confocal micro XRF imaging) of polyphase inclusions in a diamond from Guinea that formed at about 300-360 km depth reveal the existence of a deep Ca-rich source, in the absence of several common mantle minerals, like olivine, garnet and low-Ca pyroxene. This reservoir may represent metasomatized oceanic lithosphere or metamorphosed carbonaceous sediments [37].

\subsection{Three-dimensional XRF analysis of fluid inclusions in quartz}

Fluid inclusions consist of small volumes of fluid trapped and closed in a solid mineral during crystalgrowth or crack annealing. When they remained unchanged from the time of trapping, they are the only available samples of fluids preserved from rock-forming processes or subsequent fluid-rock interactions. Single inclusion analysis is commonly performed using point measurements, as well as line and image scanning using XRF and related methods. Point analysis or two-dimensional information may not be sufficient in case of the occurrence of phases of different origin below or on top of the inclusion of interest which may falsify the result. These phases include cracks, solid inclusions or other fluid inclusions. In those cases 3D information is required to study the composition of the inclusion. This is optimally done with confocal analysis as this technique allows to focus interest on the inclusions.

Documented examples of work in this area are concerned with arsenic speciation in fluid inclusions using micro-beam X-ray absorption spectroscopy [38], the study by micro-XRF of two-phase (liquid plus gas) inclusions in a gold deposit in Italy $[39,40]$ and the analysis of single fluid inclusions by combining SR induced micro-XRF, imaging, diffraction) and transmission in crustal rocks [41].

Results show that element concentrations calculated using SR XRF spectra are in relatively good agreement with those determined by crush-leach analysis and results obtained by laser ablation inductively coupled plasma mass spectrometry [41]. These results indicate that third generation SR XRS is a promising range of techniques for tracking trace metals in individual inclusions (down to the $100 \mathrm{ppb}$ level) and, hence, as an invaluable tool for reconstructing the relative chronology of paleohydrothermal events. 


\subsection{Analysis of ice cores}

The historic pollution of our environment by heavy metals is commonly assessed by analyzing a large variety of snow and ice cores from Greenland, Antarctica, the Alps, the Himalayas and the Andes for a wide range of heavy metals (especially lead, mercury, cadmium, copper, arsenic, antimony, iridium, platinum, palladium, rhodium, uranium and plutonium). This is mostly performed by the analysis of bulk ice samples originating from various ice core programmes using a range of ultra-trace analytical techniques. Amongst others, Boutron pioneered in Grenoble the development of sophisticated techniques suitable for the reliable determination of heavy metals (composition, isotopic ratios and speciation) at the incredibly low concentration levels of less than 0.1 picogram per gram at which these metals are found in Greenland and Antarctic snow and ice cores [42].

The analysis of individual particulate material is considerably less common but is now possible with SR sources.

Aeolian mineral dust archived in polar and mid latitude ice cores represents a precious proxy for assessing environmental and climatic variations at different timescales. In this respect, the identification of dust mineralogy plays a key role. Cibin et al. [43] performed XAS experiments on mineral dust particles extracted from Antarctic and from Alpine firn cores using the grazing incidence geometry at the Fe K-edge. A dedicated high vacuum experimental chamber was set up for normal-incidence and total-reflection XRF and XAS analyses on minute amounts of sample at the Stanford Synchrotron Radiation Laboratory. Results show that this experimental technique and protocol allows recognizing iron inclusion in the mineral fraction of the insoluble dust in the 1-10 microgram range [43].

\subsection{Studies of crater impacts}

The most famous and best preserved meteorite crater in the world is the Barringer Meteor Crater (Arizona, USA) which is estimated to be 50,000 years old. The chemical composition of the metallic objects (spheroids and particles) of the Barringer Crater and the "projectile" (the Canyon Diablo Meteor) has been determined by several authors. Uzonyi et al. [44,45]. presented analytical results for various impact micro-objects applying scanning electron microscopy and different scanning nuclear microprobe techniques such as micro-PIXE [30] and deuteron induced gamma-ray emission methods. The lateral distribution of elements down to carbon were investigated in minute grains of impactite slag (Fe-Si-Ni system) using SR micro-XRF. Efforts were focused on the elemental characterization of various impact materials collected from the ejecta layer of the crater. Micro-PIXE and SR micro XRF allow the identification of the impact objects and also yield information on the projectile and the impact process itself. Analytical results show compositional changes of impact materials depending on their source. Iron-Ni metals of meteoroids can be mixed with target rocks under impact condition All this results in the formation of different mixing ratios of $\mathrm{Fe}-\mathrm{Ni}-\mathrm{Co}$ system.

\subsection{Applications in environmental toxicology}

SR-based techniques have become increasingly employed in various fields of life science. There is a growing interest in the biomedical community in obtaining information concerning the distribution and local chemical environment of metals in tissues and cells [29]. We will give one characteristic example concerning imaging applications in this field.

De Samber et al. [46,47] used spatially resolved X-ray micro/nanospectroscopy for imaging a model animal (Daphnia magna) using laboratory instruments and SR sources. Daphnia magna is also known as the water flea and is a small planktonic crustacean ( $c$ a 2-5 mm in length) that can be used for the study of toxic effects caused by toxicants in water after laboratory exposure conditions. A number of different laboratory techniques were used for the experiments, in addition to 90 nanometer spatial resolution micro-XRF and XANES tomography and confocal imaging provided 3-dimensional images of the organism and information on the distribution of a number of elements at between the 10 and $100 \mathrm{ppb}$ level for a number of elements in the different structural parts of the organism. The data obtained considerably increased the information available through the use of other analytical techniques such as electron microscopy and laser ablation inductively coupled plasma mass spectrometry. 


\subsection{Applications in soil science}

The understanding of solid phase speciation of contaminants in soil is essential in terms of environmental management. Many applications in soil science now use a combinatory approach of SR based techniques such as XRF, XRD and XAS.

Accumulation of micronutrients and contaminants in plants or plant species such as seeds, speciation and localisation of metals and metalloids in hyper and non-hyper accumulator plants, soil contaminant transport, studies of the rhizosphere (the volume of soil immediately surrounding the roots), the accumulation of micronutrients and contaminants in seeds are mentioned by Lombi and Susini in a recent overview of SR-based techniques for plant and soil science [16].

Direct mercury $(\mathrm{Hg})$ speciation was assessed for soil samples of different size. $\mathrm{Hg}$ chemical forms were identified and quantified by sequential extractions and bulk- and micro-analytical techniques exploiting SR generated X-rays. In particular, micro-XRF, micro-XRD and micro-XANES were used. The main Hg-species found in the soil samples were metacinnabar (beta-HgS), cinnabar (alpha-HgS), corderoite and an amorphous phase containing $\mathrm{Hg}$ bound to chlorine and sulphur [48].

\subsection{Meteoritic ablation debris in Antarctica}

Aggregates of microscopic spherules similar in texture and composition to cosmic spherules or meteorite ablation spheres found at Miller Butte, Victoria Land, Antarctica were studied by means of electron microprobe analyses and SR XRD. Results indicate the presence of meteorite ablation debris formed during the atmospheric entry of a large meteorite of ordinary or carbonaceous chondritic composition. Comparison with the available literature data shows that the ablation debris found at Miller Butte is most likely paired with the extraterrestrial dust found in a similar to 480,000 year old ice layer in the EPICA-Dome C and Dome Fuji ice cores (East Antarctic ice sheet), thereby documenting a continental-scale distribution of ablation debris associated with a major meteoritic impact event $[49,50]$.

\subsection{Characterization of cometary matter returned by the NASA stardust mission}

Using XRF nanotomography with sub-micrometer spatial resolution, the fully three-dimensional (3D) distribution of main and trace elements from calcium to selenium was determined at a spatial resolution level of 200 nanometer within unique (circa 2 micrometer diameter) comet coma particles brought to Earth by the National Aeronautical and Space Administration (NASA) Stardust mission to Comet 81P/Wild2. The measurements were performed on terminal particles located deep within an aerogel host material (a three dimensional silicon network), which was used to capture comet and interstellar grain particles in space and in which they were preserved. In addition smaller particles deposited in the aerogel along the particle track were analyzed. Using a symmetric dual-detector arrangement a rotation angle of $180^{\circ}$ was sufficient for the full tomographic reconstruction. Dedicated correction algorithms were developed to correct for sub-micrometer-level distortions within the tomographic data set arising from instabilities of the sample positioning system during data collection [51].

Principle component analysis (PCA) was applied to two grains indicating that regions in the terminal particle and the second particle consist of minerals such as anorthite or grossite and gehlenite, monticellite or dmitryivanovite $\left(\mathrm{CaAl}_{2} \mathrm{O}_{4}\right)$, respectively. The findings demonstrate that high spatial resolution $(300 \times 300$ nanometer $)$ SR-XRF with confocal geometry and PCA analysis is capable of identifying calcium-aluminium-rich inclusions without the need to extract particles from the aerogel matrix.

These particles are thought to originate near the centre of protoplanetary disc of our solar system in the early state of its formation The SR-XRF mapping also reveals that the highest concentrations of $\mathrm{Ca}, \mathrm{Ti}, \mathrm{Fe}$ (and $\mathrm{Ni}$ ) measured within each grain belongs to different areas of the respective 3D maps which indicate that the particles are composed of several chemically diverse mineral phases [52].

These measurements underline the critical role that synchrotron microprobes play in the study of this important and technically extremely challenging Stardust Interstellar Dust Collector (SIDC) 
Table 1. composition of two particles of size $2 \times 2$ micrometer of the SIDC project (data provided by L. Vincze, Ghent University, Belgium).

\begin{tabular}{|l|l|l|}
\hline Element & Concentration, particle 1 & Concentration, particle 2 \\
\hline $\mathrm{Ca}$ & $3.64 \pm 0.06(\%)$ & $2.17 \pm 0.04(\%)$ \\
\hline $\mathrm{Ti}$ & $0.02 \pm 0.01(\%)$ & $0.15 \pm 0.01(\%)$ \\
\hline $\mathrm{Fe}$ & $0.28 \pm 0.03(\%)$ & $0.11 \pm 0.02(\%)$ \\
\hline $\mathrm{Ni}$ & $0.013 \pm 0.005(\%)$ & $0.003 \pm 0.001(\%)$ \\
\hline $\mathrm{Mn}$ & $0.03 \pm 0.01(\mathrm{ppm})$ & $0.02 \pm 0.005(\mathrm{ppm})$ \\
\hline $\mathrm{Cu}$ & $0.002 \pm 0.001(\mathrm{ppm})$ & $0.002 \pm 0.001(\mathrm{ppm})$ \\
\hline
\end{tabular}

project. A number of other SR installations X-ray microprobes were also used at the APS and the NSLS (National Synchrotron Light Source, Brookhaven National Laboratory, New York) [53].

Table I gives the results the average composition for a number of the elements in two micron sized particles detected in the particular SIDC samples studied. The data show the precision of the results which provides information to estimate the enrichment compared to the abundance of the elements in meteoritic material [54].

\section{Conclusion}

Hard XRS microscopy techniques that focus on scanning microscopy with synchrotron radiation are important in various scientific fields. Their strength compared to other microscopies rests, in the first place, with the large penetration depth of high energy X-rays in matter. This allows one to investigate the interior of an object without destructive sample preparation. Thus in combination with tomography, local information from inside of a specimen can be obtained.

Progress has been driven by the unique properties of X-ray beams produced by third generation synchrotron sources. The very low emittance coupled with high brilliance allows one to develop efficient focusing devices for new X-ray microscopy techniques. The gains in brilliance of SR sources over the past decade and advances in focusing optics during the same period provide now spatial resolution at the sub-micrometer range while maintaining a high sensitivity and the 10 nanometer sample size range is expected in the near future [55,56].

During the past decade, applications of SR methods in many fields of science, ranging from physics and chemistry to materials, geo-, and environmental science, biomedicine, and nanotechnology. Applications have grown significantly in 3D imaging analysis of materials with XRF microtomography at the microscopic and the sub-microscopic level. In addition, the use has expanded in molecular-level speciation applications of environmental materials to study fundamental chemical and biological processes with application of XAS spectroscopy and related SR methods, including microXANES, micro-XRD and photoemission spectroscopy.

\section{References}

1. R.E. Van Grieken, A. Markowicz, (editors), Handbook of X-ray Spectrometry, 2nd edition (Dekker, New York) (2002)

2. Kouichi Tsuji, Kazuhiko Nakano, Yoshio Takahashi, Kouichi Hayashi, Chul-Un Ro, Analytical Chemistry, 82, 4950 (2010)

3. http://www. lightsources.org

4. Freddy Adams, Koen Janssens, Erca II - Les Ullis, Éditions de Physique, 183 (1996)

5. Koen Janssens, Freddy Adams, Anders Rindby, Microscopic X-ray fluorescence analysis (Wiley, Chichester) (2000) 
6. Freddy Adams, X-ray fluorescence and emission-synchrotron X-ray fluorescence, Encyclopedia of analytical science, 9, 458, Paul J. Worsfold, (editor) (Elsevier, Oxford) (2005)

7. L.Vincze, K. Janssens, F. Adams, L. Vincze, K. Janssens, F. Adams, X-Ray Spectrometry, 24, 27 (1995)

8. H. Mimura, S. Handa, S.T. Kimura, H. Yumoto, D. Yamakawa, H. Yokoyama, S. Matsuyama, K.Inagaki, K. Yamamura, Y. Sano, K. Tamasaku, Y Nishino, M. Yabashi, T. Ishikawa, K. Yamauchi, Nature Physics, 6, 57 (2010)

9. P. Van Espen, H. Nullens, F. Adams, Nuclear Instruments and Methods in Physics Research: B: beam interactions with materials and atoms, 142, 243 (1977)

10. L. Vincze, K. Janssens, B. Vekemans, F. Adams, Spectrochimica Acta, B, atomic spectroscopy, 54, 1711 (1999)

11. L. Vincze, K. Janssens, B. Vekemans, F. Adams, Journal of Analytical Atomic Spectrometry, 14, 529 (1999)

12. A. Simionivici, M. Chukalina, M.C. Schroer, M. Drakopoulos, A. Snigirev, I Snigireva, B. Lengerer, K. Janssens, F. Adams, IEEE Transactions Nuclear Science, 47, 2736 (2000)

13. Koen Janssens, Wout De Nolf, Geert Van Der Snickt, Laszlo Vincze, Bart Vekemans, Roberto Terzano, Frank E. Brenker, Trends in Analytical Chemistry, 29, 464 (2010)

14. B. Vekemans, L. Vincze, F.E. Brenker, F. Adams, Journal of Analytical Atomic Spectrometry, 19, $1302(2004)$

15. L. Vincze, B. Vekemans, F.E. Brenker, G. Falkenberg, K. Rickers, A. Somogyi, M. Kersten and F. Adams, Analytical Chemistry, 76, 6786 (2004)

16. E. Lombi, J. Susini, Plant and Soil, 320, 1 (2009)

17. A. Prange, H. Schwenke, Advances in X-ray Analysis, 35, 899 (1992)

18. F. Meirer, A. Singh, G. Pepponi, C. Streli, T. Homma, P. Pianetta, Trends in Analytical Chemistry, 29, 479 (2010)

19. C. Streli, G. Pepponi, P. Wobrauschek, C. Jokubonis, G. Falkenberg, G. Zaray, J. Broekaert, U. Fittschen, B. Peschel, Spectrochimica Acta, B, atomic spectroscopy, 61, 1129 (2006)

20. A. Rindby, P. Engstrom, K. Janssens J. Osan, Nuclear instruments and methods in physics research, B: beam interactions with materials and atoms, 124, 591 (1997)

21. Trina Bouvet, Carsten Detlefs, Edward Mitchell, Jean-Luc Revol, ESRF Science and Technology Programme 2008-2017, (ESRF, Grenoble) (2007)

22. W. Yue, G.L. Zhang, P. Liu, J. Sun, Y.K. Hwu, J. Je, M.G. Tan, Y. Li, Nuclear Instruments and Methods in Physics Research, B: beam interactions with materials and atoms, 262, 304 (2007)

23. A. Somogyi, F. Polack, T. Moreno, AIP Conference Proceedings, 1234, 395 (2010)

24. http://ec.europa.eu/research/infrastructures/index_en. cfm?pg=esfri

25. P. Tafforeau , R. Boistel, E. Boller, A. Bravin, M. Brunet, Y. Chaimanee, P. Cloetens, M. Feist, J. Hoszowska, J.J. Jaeger, R.F. Kay, V. Lazzari, L. Marivaux, A. Nel, C. Nemoz, X. Thibault, P. Vignaud, S. Zabler, Applied Physics, A: materials science \& processing, 83, 195 (2006)

26. Geert Silversmit, Bart Vekemans, Sergey Nikitenko, Sylvia, Schmitz, Tom Schoonjans, Frank E. Brenker, Laszlo Vincze, Phys. Chem. Chem. Phys., DOI: 10.1039/c004103n (2010)

27. A. Somogyi, M. Drakopoulos, L. Vincze, B. Vekemans, C. Camerani, K. Janssens, A. Snigirev, F. Adams, X-ray Spectrometry, 30, 242 (2001)

28. A. Somogyi, M. Drakopoulis, B. Vekemans, L. Vincze, A. Simionovici, F. Adams, Nuclear instruments and methods in physics research, B: beam interactions with materials and atoms, 199, 559 (2003)

29. R.A. Barrea, D. Gore, N. Kujala, C. Karanfil, S. Kozyrenko, R. Heurich, M. Vukonich, R. Huang, T. Paunesku, G. Woloschak, G.T.C. Irving, Journal of Synchrotron Radiation, 17, 522 (2010)

30. Z. Kertesz, Z. Szikszai, Z. Szoboszlai, A. Simon, R. Huszank, I. Uzonyi, Nuclear Instruments and Methods in Physics Research, B: beam interactions with materials and atoms, 267, 2236 (2009)

31. L. Vincze, A. Somogyi, J. Osán, B. Vekemans, S. Török, K. Janssens, F. Adams, Analytical Chemistry, 74, 1128 (2002)

32. B. Salbu, Journal of Environmental Radioactivity, 96, 47 (2007)

33. B. Salbu, T. Krekling, O.C. Lind OC, D.H. Oughton, M. Drakopoulos, A. Simionovici, I. Snigireva, A. Snigirev, T. Weitkamp, F. Adams, K. Janssens, V.A. Kashparov, Nuclear Instruments 
and Methods in Physics Research, Section A, accelerators, spectrometers, detectors and associated equipment 467, 1249 (2001)

34. O.C. Lind, B. Salbu, L. Skipperud, K. Janssens, J. Jaroszewicz, W. De Nolf, Journal of Environmental Radioactivity, 100, 301 (2009)

35. O.C. Lind, B. Salbu, K. Janssens, K. Proost, H. Dahlgaaard, Journal of Environmental Radioactivity, 81, 21 (2005)

36. C. Lerouge, F. Claret, M.A. Denecke, G. Wille, G. Falkenberg, C. Ramboz, C. Beny, E. Giffaut, T. Schaefer, E.C. Gaucher, C. Tournassat, Physics and Chemistry of the Earth, 35, 271 (2010)

37. F.E. Brenker, L. Vincze, B. Vekemans, L. Nasdala, T. Stachel, C. Vollmer, M. Kersten, A. Somogyi, F. Adams, W. Joswig, J.W. Haris, Earth and Planetary Science Letters, 236, 579 (2005)

38. J. Smith, J. Cauzid, D. Testemale, W. Liu, J.L Hazemann, O. Proux, B. Etschmann, P. Philippot, D. Banks, P. Williams, J. Brugger, American Mineralogist, 95, 921 (2010)

39. P. Philippot, A. Somogyi, B. Menez, A. Simionovici, P. Bleuet, Chemical Geology, 227, 165 (2006)

40. J. Cauzid, P. Philippot, A. Somgyi, A. Simionovici, P. Bleuet, Analytical Chemistry, 76, 3988 (2004)

41. P. Philippot, B. Menez, M. Drakopoulos, A. Simionovici, A. Snigirev, I. Snigireva, Chemical Geology, 173, $151(2001)$

42. F.A.M Planchon, C.F. Boutron, C. Barbante, G. Cozzi, V. Gaspari, E.W. Wolff, C.P Ferrari, P. Cescon, Earth and Planetary Science Letters , 200, 207 (2002)

43. G. Cibin, A. Marcelli, V. Maggi, M. Sala, F. Marino, B. Delmonte, S. Albani, S. Pignott Spectrochimica Acta, Part B, atomic spectroscopy,63, 1503 (2008)

44. I. Uzonyia, Gy. Szoör, B. Vekemans, L. Vincze, P. Rozs, Gy. Szabo, A. Somogyi, F. Adams, A.Z. Kiss, Spectrochimica Acta, B, atomic spectroscopy, 59, 1717 (2004)

45. I. Uzonyi, Gy Szöör, P. Rozsa, B.Vekemans, L. Vincze, F. Adams, M. Drakopoulos, A. Somogyi, A.A.Z. Kiss, Nuclear Instruments and Methods in Physics Research: B: beam interactions with materials and atoms, 219/220, 555 (2004)

46. B. De Samber, Spatially resolved X-ray Micro/Nanospectroscopy and imaging on the model organism Daphnia magna using laboratory and synchrotron sources, Ph.D. thesis, Ghent University, Belgium (2010)

47. B. De Samber, G. Silversmit, R. Evens, K. De Schamphelaere, C. Janssen, B. Masschaele, L Van Hoorebeke., L. Balcaen, F. Vanhaecke, L. Vincze, Anaytical and Bioanalytical Chemistry, 390, 267 (2008)

48. Roberto Terzano, Anna Santoro, Matteo Spagnuolo, Bart Vekemans, Luca (Medici, Koen Janssens, Joerg Goettlicher, Melissa Denecke, Stefan Mangold, Pacifico Ruggiero, Environmental Pollution, 158, 2702 (2010)

49. M. van Ginneken, L. Folco, N. Perchiazzi, P. Rochette, P.A. Bland, Earth and Planetary Science Letters, 293, 104 (2010)

50. Urs Ruth, Carlo Barbante, Matthias Bigler, Barbara Delmonte, Hubertus Fischer, Paolo Gabrielli, Vania Gaspari, Patrik Kaufmann, Fabrice Lambert, Valter Maggi, Frederica Marino, Jean-Robert Petit, Roberto Udisti, Dietmar Wagenbach, Anna. Wegner, Eric Wolff, Environmental Science \& Technology, 42, 5675 (2008)

51. Geert Silversmit, Bart Vekemans, Frank E. Brenker, Sylvia Schmitz, Manfred Burghammer, Christian Riekel, Laszlo Vincze, Analytical Chemistry, 81, 6107 (2009)

52. Sylvia Schmitz, Frank E Brenker, Tom Schoonjans, Bart Vekemans, Geert Silversmit, Laszlo Vincze, Manfred Burghammer, Christian Riekel, Geochimica Cosmochimica. Acta, 73, 5483 (2009)

53. A.J. Westphal... M.E. Zolensky, M.E., X-Ray Optics and Microanalysis Proceedings, AIP Conference Proceedings, 1221, 131 (2010)

54. E. Anders, N. Grevisse, Geochimica Cosmochimica Acta, 53, 197 (1989)

55. A. Snigirev, I. Snigireva, Comptes Rendus Physique 9, 507 (2008)

56. José Baruchel, Pierre Bleuet, Alberto Bravin, Paola Coan, Enju Lima, Anders Madsen, Wolfgang Ludwig, Petra Pernot, Jean Susini, Comptes Rendus Physique 9, 624 (2008) 